\title{
Un silence assourdissant à la césure : les guerres larvées de l'e caduc entre odipiens, misogynes et glottophobes
}

\author{
DAVID MOUCAUD \\ Université Paris 3
}

Largement étudié sous l'angle "naturel " d'une tabula rasa générationnelle, le changement technique, voire formaliste, connu comme "l'abolition de la coupe féminine " vers 1515 est un noud conflictuel bien plus sinueux qu'il n'y paraît de prime abord. Ce silence formel, imposé à la césure par de jeunes poètes (Marot) et de moins jeunes (Lemaire), fit grand bruit une fois brandi comme fer de lance misogyne contre une mise aux normes linguistique plus insidieusement autoritaire. Outre le faux débat de la modernité qui en rejoue un plus ancien, outre celui de la misogynie qui littéralise incongrûment une affaire des plus techniques, les derniers feux des coupes "épique " et "lyrique "semblent aussi devoir masquer la dissidence d'une spécificité phonétique régionale contre la francisation centraliste de la chose poétique, sous l'effet d'un nouvel unanimisme de cour qui, discrètement, oppose comme rarement l'ordre à une rébellion réactionnaire.

Widely studied from the "natural" perspective of a generational tabula rasa, the formalist shift in poetic technique known as the abolition of the coupe feminine, which occurred around 1515, is a thorny issue much more complicated than it would first appear. This formal silence, imposed upon the caesura by young poets (Marot) and older ones (Lemaire) alike, caused a sensation once wielded as a misogynist spearhead against a more insidiously authoritarian linguistic normalization. Beyond the false debate around modernity that re-enacts an older one, and beyond the debate, conducted in misogynist terms, that incongruously literalizes a highly technical issue, the last flickers of "epic" and "lyrical" coupes would seem also to need to conceal the dissidence of phonological, regional specificity against the centralist francisation of the "poetic thing," under the influence of a new unanimity at the Court of Valois that discretely — and unusually -opposes order to reactionary rebellion.

U n changement technique, de premier plan mais néanmoins aussi discret et (apparemment) consensuel que celui qu'on a appelé « l'abolition de la coupe féminine ${ }^{1}$ est-il soluble dans la querelle et la construction conflictuelle des publics, sur lesquelles nous sommes ici appelé à nous interroger?

1. Léon Émile Kastner, "Les grands rhétoriqueurs et l'abolition de la coupe féminine ", Revue des langues romanes 46 (1903) : 289-297 ; Georges Lote, Histoire du vers français, éd. Joëlle Gardes-Tamine, Jean Molino et Lucien Victor, 9 vols. (Paris, Aix : Boivin — Presses universitaires de Provence, 1988 [1949]), 4-2 ; Giovanna Bellati, "Bouchet e l'abolizione della "coupe féminine" ", Studi francesi 44 (1988) : 73-81; Thierry Mantovani, "Gratien du Pont et l'abolition de la césure féminine », in Grands rhétoriqueurs, Cahiers V.-L. Saulnier 14 (Paris : Presses de l'École Normale Supérieure, 1997), 55-74. 
Au siècle pourtant le plus rompu à la dissertatio, elle fit couler, semble-til, moins d'encre que ne devait le faire quelques siècles plus tard son pendant capillo-révolutionnaire : la coupe " à la garçonne ». S’il est, comme l'assure Georges Lote, "bien peu [de techniciens du XVI ${ }^{\text {e }}$ siècle] qui négligent d'en parler », on doit s'interroger sur "l'ardeur peu commune » que selon lui " ils mettent à [...] proscrire [un $e$ muet quelconque qui ne pourrait être mangé par l'élision] $»^{2}$, tant ce changement paraît irrévocable au plan poétique, souffrant peu d'exceptions et moins de réactions encore. Il n'est que de lire à cet égard Sébillet, en 1548, au sujet de cette " couppe fémenine, laquéle non observée dés anciens, ne de Marot en son jeune eage [...] toutesfois est aujourd'huy gardée inviolablement par tous les bons Poëtes de ce temps $»^{3}$. Cette coupe " inviol[ée] » rejoint la catachrèse qui depuis un siècle ${ }^{4}$ sexualise alors le rapport entre le genre des mots et l'inexpugnable forteresse féminine. On notera toutefois la curiosité voulant que " garder inviolablement » la coupe féminine revienne tout bonnement à l'éviter ou à la réduire - symboliquement - au silence de la synérèse.

Dolet désigne pourtant ce changement - l'abolition - à la manière d'une rupture politique ${ }^{5}$, par contagion peut-être avec le nom de son objet - la césure - dont la connotation, sortie du champ poéticien, n'est pas moins conflictuelle $^{6}$. Quelles tensions, quelle portée polémique l'abolition des césures

\section{Lote, Histoire du vers français, $4: 23$.}

3. Thomas Sébillet, Art poëtique françoys (1548), éd. Francis Goyet, Traités de poétique et de rhétorique de la Renaissance (Paris : Librairie générale française, 1990), 74 (nous restituons systématiquement les graphies originales d'après l'éd. Félix Gaiffe [Paris : Édouard Cornély, 1910]).

4. Si Ernest Langlois (Recueils d'arts de seconde rhétorique [Paris : Imprimerie Nationale, 1902], lxvi ; 3) rappelle que cette analogie sexualisante est déjà bien présente dans l'Archiloge Sophie de Jacques Legrand, antérieur à 1400, il est frappant de constater que L'Art de dictier d'Eustache Deschamps, contemporain de L'Archiloge, se préoccupe bien plus de consonnes que de voyelles, et ne les affecte en rien de qualités anthropomorphes... Voir l'éd. Gaston Raynaud, Euvres complètes d'Eustache Deschamps (Paris : Firmin-Didot, 1891), 272-273.

5. La métaphore de l'« abolition » est sans doute passée par Étienne Dolet, La Manière de bien traduire d'une langue en autre davantage De la Punctuation de la Langue françoyse puis Des Accents d'ycelle (Lyon : Étienne Dolet, 1540), 38 : «Et les Faictistes, appellent cela couppe feminine, c'est à dire abolition de l'e feminin, qui rencontre une aultre voyelle, par laquelle il est aboli apres la quatriesme syllabe du vers ».

6. Peut-on envisager une réminiscence érudite dans l'étonnante projection conflictuelle de l'histoire littéraire sur les sociétés modernes, qui fit connaître naguère sous le nom de "bataille de la césure " 
féminines peut-elle bien traduire, et en quoi programme-t-elle une divergence entre des corps de lecteurs? Tel est le double problème que nous nous proposons d'examiner ici. Il est avant tout méthodologique, en ceci qu'il se conçoit moins du point de vue de son objet que comme un angle sous lequel il s'agirait de le réexaminer.

Un premier axe est donné par l'histoire de la poésie et de la versification, qui s'accorde pour dater assez précisément cette disparition : entre 1512 et 1518, pour les bornes les plus généreuses. Lote signale que le Puy de Rouen récompense à partir de 1516 le "meilleur chant royal [...] sans coupes feminines, s'ilz ne sont synalymphes $»^{7}$. Il y aurait bien sûr un amont à cette disparition, que Kastner fait remonter à Chastelain et Molinet dans les années $1480^{8}$; elle connaît aussi en aval une certaine résistance, dont Gratien du Pont de Drusac en 1534 est un fervent représentant.

Technique et daté, le phénomène ne paraît pas, per se, justifier le bruit ni la fureur. Nous en avons d'emblée mentionné la discrétion, qu'il faut étendre à tous les sens qu'on peut donner à ce mot. Discret, il l'est au sens mathématique, étymologique, en tant qu'il est distinctif et très circonscrit - on dirait aujourd'hui localisé ; il l'est encore doublement, parce qu'il ne fait pas de bruit: non seulement renoncer aux césures féminines consiste précisément à étouffer des voyelles muettes, mais contrairement, par exemple, au recours ou non à la rime équivoquée, les textes ne le thématisent guère ; il l'est enfin, dans ce sens qui nous est un peu ancien, qu'il semble avisé, voire de bon sens : le grand telos de la versification semble donner raison à cet arraisonnement d'une mesure moins permissive où le demi-vers a une cadence et une seule 9 .

\footnotetext{
un épisode de la récente histoire de la Bibliothèque nationale de France ? Voir François Devinat, « Dix ans d'affrontements pharaoniques ", Libération, 7 octobre 1998 et François Stasse, "Vieille dame, grande dame : la Bibliothèque nationale de France », communication devant l'Académie des Sciences morales et politiques, Paris, 2003, http://www.canalacademie.com/ida120-Vieille-dame-grande-damela-Bibliotheque-nationale-de-France.html.

7. Lote, Histoire du vers français, $4: 24$. Comprendre : si elles ne sont élidées par «synalèphe », épousant alors la voyelle subséquente.

8. Kastner, «Les grands rhétoriqueurs », 290.

9. Philippe Martinon, «Études sur le vers français. La genèse des règles de Jean Lemaire à Malherbe ", Revue d'histoire littéraire de la France 16 (1909) : 62, explique : « [...] accompagnement obligé d'une éducation de plus en plus raffinée de l'oreille».
} 
Qu'il s'agisse de ce qui se passe à la césure ou à la rime (avec ce qu'on appellera bientôt son alternance en genre, qui se règle dans les mêmes années), ou encore dans l'accord des participes (tocade italianisante de Marot pour amuser la Cour, semble-t-il), une part conséquente de la mise aux normes poétique du début du $\mathrm{XVI}^{\mathrm{e}}$ siècle tourne autour de la voyelle finale -e que l'on dit « féminine ${ }^{10}$, muette, caduque dès lors qu'elle est vouée au silence.

L'enjeu est de taille et nourrit un second axe de réflexion : le peu qui soit donné à lire alors sur la question est effectivement tourné - ou faudraitil dire détourné - pour ou contre la gent féminine. Alors que la chose est purement technique, on la corrèle au grand débat misogyne - moral et, partant, littéraire - de la Querelle des femmes ${ }^{11}$. Ce qu'on ne fait pas du tout au sujet de la rime équivoque, volontiers virile pourtant, voire viriliste au regard des calembours qu'elle motive ${ }^{12}$. Ni les vices supposés féminins, ni un casus belli comme la "féminisation des noms de métiers » ne sont à l'œuvre, mais seulement le dépérissement phonétique et poétique d'un - $e$ final de la vieille langue, arbitrairement prononcé ou non parce qu'il est à une position particulière du vers et ainsi susceptible d'être apocopé (en " apostrophe ») devant une consonne, ou au contraire prononcé en hiatus (sans "synalèphe ») devant une voyelle. De même que la défense de l'alternance en genre et peutêtre l'accord du participe passé à l'italienne, que transpose l'invention du damier équitablement noir et blanc, de même tendrait-on à canaliser l'arbitraire du signe dans un sens (d'une réduction à la nullité) ou dans l'autre (d'une

10. Le même volume d'Étienne Dolet, La Manière de bien traduire, 34-37, développe la théorie, déjà ancienne, qui sous-tend cette conception sexualisée du genre grammatical : "La lettre appellée $e$ a double son et prolation en Françoys : la premiere est dicte masculine et l'aultre feminine. La masculine est nommée ainsi, pour ce que é, masculin, a le son plus viril, plus robuste et plus fort sonnant [...] L'autre pronunciation de ceste lettre $e$ est feminine, c'est à dire de peu de son et sans vehemence [...] elle est de si peu de force que tousjours elle est mangée, s'il s'ensuict apres elle ung mot commencant par voyelle »; Sébillet le reprendra avec la coquetterie de l'indéfini, Art poëtique françoys, éd. Francis Goyet, 68-69 (nous soulignons) : « [il est] appellé masculin, a cause de sa force, et ne say quéle virilité qu’il ha plus que le fémenin » (68), ce dernier étant dit « tant éfféminé, qu’il ne peut oublier sa mollesse » (69).

11. Voir Éliane Viennot, "Revisiter la "Querelle des femmes” : mais de quoi parle-t-on ? ", in Revisiter la “Querelle des femmes”. Discours sur l'égalité / inégalité des sexes, de 1750 aux lendemains de la Révolution, éd. Éliane Viennot, avec la collaboration de Nicole Pellegrin (Saint-Étienne : Publications de l'Université de Saint-Étienne, 2012), 7-29.

12. Voir François Cornilliat, "Quelques enjeux de la rime équivoque », Bulletin de l'Association d'étude sur l'Humanisme, la Réforme et la Renaissance 33 (1991) : 5-30. 
valorisation paritaire). Puisqu'à la faveur de la Querelle, se joue clairement un conflit de domination et de légitimité, ce locus technicus de la césure est investi d'une charge critique flattant préférentiellement un lectorat féministe ou antiféministe dont le texte versifié prétend nourrir l'arbitrage.

Un dernier fil s'impose à cette enquête, que déroule une divergence phonétique : comme Kastner l'entrevoit le premier, cette rationalisation suit un mouvement du Nord vers le Sud, qui pourrait bien motiver linguistiquement, d'une manière plus complexe qu'il n'y paraît au premier regard, les derniers feux de la coupe féminine. Cette fois, la progression différenciée d'une langue littéraire royale et surtout les effets sur elle de dictions diatopiquement déviantes seraient en cause. C'est alors une autre forme de séduction ou de conformation à son public (et l'agression d'un public contraire) que pourrait viser le versificateur, soucieux de faire adhérer à la (non) déclamation des finales féminines que programme inéluctablement un mètre, des lecteurs pour qui il serait moins naturel de les élider ou de les amuïr.

\section{De l'éloquence des muettes}

Rappelons en quelques lignes quelle est la teneur de ce discret changement dans l'ars versificandi, en empruntant nos exemples à Bouchet, qui a pratiqué ces coupes avant de spectaculaires repentirs.

La césure du décasyllabe ou de l'alexandrin partage ces vers composés en deux mesures. De même que la rime, qui termine la seconde et le vers, peut être prolongée d'une syllabe surnuméraire par un -e post-tonique, une coupe féminine vient de même altérer la netteté de la césure, suivant deux modalités contradictoires.

À l'imitation de la rime féminine, la césure épique enfreint le calcul des positions métriques en maintenant une syllabe surnuméraire - qui ne fait pas position - après la quatrième position du décasyllabe ou la sixième de l'alexandrin :

Gasteurs de fill(es) + sont ces folz escoliers ${ }^{13}$

13. Jean Bouchet, Les Regnars traversant les perilleuses voyes des folles fiances du monde (Paris : Antoine Vérard, vers 1503), fol. A3 $\mathrm{r}^{\circ}$; nous empruntons aux ouvrages des métriciens depuis Benoît de Cornulier la notation commode voyant délimiter les hémistiches par le signe « + », et plaçons entre parenthèses la syllabe qui échappe au décompte métrique. 
Si cet - e caduc final amorce cependant un second hémistiche débuté par une voyelle, alors l'infraction est minimisée par la synalèphe : un mot déborde la césure, sans cependant qu'une syllabe soit de trop dans le vers, en un avatar de l'enjambement « à l'italienne ${ }^{14}$ :

De la justi(ce) + helas certainement ${ }^{15}$ (ou : De la justi- + -ce helas certainement)

La césure lyrique impose quant à elle à la quatrième position du décasyllabe ou à la sixième de l'alexandrin une syllabe numéraire, comme elle le serait devant une consonne à une autre place dans le vers, tenue par un - e caduc :

\section{Toutes prestes + de meurdrir ces meschans ${ }^{16}$}

Il arrive qu'une telle finale caduque doive faire position, à cette place, devant une voyelle (lorsqu'ailleurs elle serait élidée par synalèphe) :

J'ay veu monstre + aussi vil que chymere ${ }^{17}$

Les mots de Pierre Fabri sont peut-être plus clairs lorsqu'il proscrit ces deux possibles déséquilibres du vers dans la composition du Chant royal : « [...] termination feminine ne faict point pleine syllabe [... et] syllabe feminine a la IIII. place n'est que de trois et sa passe, qui est diminution de couppe, ou elle est de quattre et sa passe, qui est addition ${ }^{18}$.

Rien donc que de très technique, de prime abord, et qui résulte de singularisations anciennes dans l'histoire de la versification, autorisant jusqu'aux renoncements des années 1480-1510 ces différentes configurations :

14. Léon Émile Kastner, A History of French Versification (Oxford : Clarendon, 1903), 83-84.

15. Bouchet, Regnars, fol. A2 v.

16. Bouchet, Regnars, fol. A3 vo.

17. Bouchet, Regnars, fol. A2 vo.

18. Pierre Fabri, Le grand et vray Art de pleine rhetoricque (1521), éd. Antoine Héron, 2 vols. (Paris : Société des Bibliophiles Normands, 1889-1990), 2 : 97. Il faut rappeler avec Denis Hüe, qui le cite, que Fabri est juge au Puy de Rouen : Denis Hüe, La Poésie palinodique à Rouen, 1486-1550 (Paris : Honoré Champion, 2002), 897-899. 
ordinaire

épique

(épique "à l'italienne»)

lyrique

(lyrique sans synalèphe)
$\mathrm{CV} \mathrm{CV} \mathrm{CV} \mathrm{CV}_{4}+$ $\mathrm{CV} \mathrm{CV} \mathrm{CV} \mathrm{CV}_{4}(\mathrm{Ce}(s / n t))+$ $\mathrm{CV} \mathrm{CV} \mathrm{CV} \mathrm{CV}_{4}(\mathrm{Ce})+$

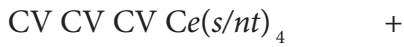
$\mathrm{CV} \mathrm{CV} \mathrm{CV} \mathrm{Ce} e_{4}$
CV CV CV CV CV CV CV CV CV CV CV CV $\mathrm{V}$ CV CV CV CV CV CV CV CV CV CV CV $\mathrm{V} C \mathrm{CV}$ CV CV CV

Les coupes féminines et leur abandon relèvent d'un réglage métrique (c'est-à-dire à la fois d'ajustements techniques et leur mise en règle(s) - en fait de normalisation comme de grammatisation), dont l'application est quasiment unanime vers 1510. Quand bien même il pourrait y avoir un débat de techniciens, on ne voit donc pas immédiatement en quoi un lectorat serait impliqué de manière polémique, puisque ni les causes ni les effets ne semblent d'abord conflictuels. Le public normand que décrit Denis Hüe serait certes formellement et thématiquement très conservateur ${ }^{19}$, mais un procédé progressivement tombé en désuétude ne paraît à l'inverse guère subversif.

Lorsqu'elle revient en force cependant, la question semble être un lointain prétexte cratyléen pour la défense ou la condamnation (au silence) des femmes, et masque assurément des tensions plus larvées. Aussi nous faut-il pister les traces de conflit et de destination dans ce changement poétique a priori technique et inoffensif. Elles sont essentiellement de deux ordres, qui ont leurs ramifications, et qui organiseront cette enquête : générationnel et symbolique.

En fait de désigner un «silence », il ne s'agit bien sûr pas de revenir sur la querelle de la métrique accentuelle, ni même de nous opposer frontalement à Lote lorsqu'il insiste à refuser celui d'une pause à la césure (ou à la rime ${ }^{20}$. Le silence que nous signalons est plutôt symbolique ; discrètement ou sous le bruit du faux-débat, c'est celui des causes, entre une unifiante ratio metrica et peutêtre un arasement linguistique, que Lote entrevoit lui-même dans les traités (après Deimier) : "Puis c'est le silence, car la règle est devenue définitive et ne souffre plus d'infractions $»^{21}$.

Dans l'un et l'autre cas, se joue un arraisonnement qui est bel et bien une reductio ad silentium, à la fois de cette voyelle médiévale « de trop » (selon qu'elle

19. Hüe, La Poésie palinodique, 905.

20. Lote, Histoire du vers français, $1: 84,132,146,193$; $4: 15-19$.

21. Lote, Histoire du vers français, $4: 24$. 
est perçue comme divergente, par trop féminine ou par trop provinciale...) dans un canon métrique qu'on entend rénover, des générations précédentes qu'elle vieillit instantanément et des dissidences qu'elle méprise. Du jeunisme réactionnaire, en somme, comme l'histoire joue à en répéter souvent les exemples et qui, de même, dit rarement son nom.

\section{Archaïsme de ces coupes : \\ Marot et Lemaire dos à dos, Bouchet contre lui-même}

Sans manifeste ni couronne, on observe objectivement que le tournant des années 1510 voit disparaître la coupe féminine chez les poètes. L'histoire littéraire, qui aime à voir en Clément Marot un irréfragable rénovateur, s'est longtemps contentée du repentir de jeunesse à l'égard de ces coupes, par lequel il rend hommage à son maître Jean Lemaire de Belges. Sans contester que Marot en soit, ce faisant, devenu le héraut, Kastner souligne cependant que le premier à avoir massivement délaissé la césure lyrique n'est pas Lemaire mais Cretin ${ }^{22}$. $\mathrm{Au}$ prix de corrections dont on a parfois la trace, les contemporains renoncent, à de rares exceptions, aux deux types de coupes féminines.

La question a fondé une véritable niche critique qui va de Kastner à Thierry Mantovani, en passant par Martinon, Lote, Kathleen Chesney, Claude Thiry, Jennifer Britnell, Giovanna Bellati, et Jacques Roubaud.

Jennifer Britnell a ainsi fait observer que Bouchet corrigeait ses césures lyriques entre les éditions de 1512 et 1525 de la Déploration de l'église militante ${ }^{23}$, mais n'en donne pas le compte et n'évoque pas les césures épiques. Il s'est également corrigé sur les Regnars traversant les perilleuses voyes des folles fiances $d u$ monde entre les deux états que nous en connaissons, de l'édition Vérard de 1502-1503 au manuscrit remanié de $1531^{24}$. Le changement est radical : les 36 césures lyriques et 44 césures épiques, pratiquement toutes retouchées, frappaient d'une coupe féminine un décasyllabe sur cinq. Les

22. Kastner, "Les grands rhétoriqueurs " (297), contesté par Chesney (Guillaume Cretin, Euvres poétiques, éd. Kathleen Chesney [Paris : Firmin-Didot, 1932], «Introduction », liv-lvi), qui signale au moins deux œuvres de jeunesse où les césures respectivement lyrique et épique sont expérimentées dans de grandes proportions.

23. Jean Bouchet, Deploration de l'Eglise militante, éd. Jennifer Britnell (Genève : Droz, 1991).

24. Ce dernier, conservé à la Bibliothèque municipale de Poitiers (ms. 440, 195 f.), fait actuellement l'objet d'un travail d'édition, mené par Nathalie Dauvois, Pierre Martin et Jonas Kurscheidt. 
trois vers de Bouchet précédemment donnés en exemple subissent ainsi divers déplacements syntaxiques, ou de simples substitutions lexicales, qui y suffisent:

$$
\text { imprimé Vérard (vers 1503) }
$$

Épique Gasteurs de fill(es) + sont ces folz escoliers

Lyrique Toutes prestes + de meurdrir ces meschans

Lyr. sans syn. J'ay veu monstre + aussi vil que chymere ms. remanié25 (vers 1531)

Violateurs + sont ces folz escoliers

Pour devorer + ces

malheureux marchans

Ung monstre ay veu + plus

vil qu'une chymere

\section{Un ornement désuet et imprécis}

Ce qui à la fin du $X^{e}$ siècle était encore, à la cour de France du moins, un ornement voire une ficelle de la variatio metrica, tombe soudain en disgrâce. On peut s'étonner de la rapidité du changement, puisqu'il s'opère en moins de dix ans et que les positions de chacun sont plus variées qu'il n'y paraît à considérer seulement la disparition pure et simple, sous ses deux formes (assez différentes), du phénomène. Roubaud les résume :

Certains (Jean Lemaire, par exemple) penchaient visiblement pour l'abandon de la seule césure épique. D’autres (Cretin, par exemple) pour le seul renoncement à la césure lyrique. Certains, sans proscrire absolument aucune d'entre elles, tendaient à en favoriser une au détriment de l'autre (Octovien de Saint-Gelais, la lyrique ; Jean Marot, l'épique). ${ }^{26}$

L'obligeance de Clément Marot à dire qu'il doit à Lemaire le remaniement de ses césures de jeunesse dans sa traduction de Virgile est bien connue ${ }^{27}$.

25. Bouchet, Regnars, ms. 440, respectivement f. 5 ro 7 ro $3 \mathrm{v}^{\mathrm{o}}$.

26. Jacques Roubaud, Impressions de France. Incursions dans la France du premier XVI e siècle (1500-1550) (Paris : Hatier, 1991), 109.

27. François Rigolot, " "De peu assez" : Clément Marot et Jean Lemaire de Belges », in Clément Marot "Prince des poëtes françois » : 1496-1996, actes du Colloque de Cahors, éd. Gérard Defaux et Michel 
Roubaud relève quant à lui la citation du même Lemaire par Bouchet en 1515 - et il ne résiste pas à y voir le « Marignan de la césure ${ }^{28}$ :

Et ay gardé une reigle qui veult en rithme de dix piedz la premiere partie de la ligne, si elle est de rithme imparfaicte, estre de cinq piedz, et sinalimphée avec le surplus. Ce que j’ay veu seulement garder et observer à Maistre Jehan Le Maire, indiciaire orateur moderne [nous soulignons], et non à Maistre Alain Charretier, aux Grebans, Georges, Castel, Meschinot, Millet, Maistre Octovyan de Saint Gelais, ne a plusieurs aultres bons orateurs en rithme, les euvres desquelz j'ay tousjours veu bien extimer. ${ }^{29}$

Il semble bien que l'illustre Lemaire soit communément salué comme un législateur en matière de césures épiques. Il faut encore noter que Marot ne s'est laissé aller à aucune césure lyrique dans son " églogue », alors que, trente ans après la bataille, il s'en autorise « avec malice », d'après François Rigolot ${ }^{30}$, dans la complainte de Guillaume Preudhomme de 1543.

Marot se corrige donc (avec une certaine tolérance pour les finales en -ée et en oubliant deux vers ${ }^{31}$ ), même si son revirement est moins spectaculaire et massif que celui de Bouchet. Rigolot centre quant à lui sur ce phénomène ce qu'il

Simonin (Paris : Honoré Champion, 1997), 185-199.

28. Roubaud, Impressions de France, 101. L'expression sera encore relevée par François Cornilliat, «La Complainte de Guillaume Preudhomme, ou l'adieu de Marot à la "Grande Rhétorique" ", in Clément Marot " Prince des poëtes françois » : 1496-1996, actes du Colloque de Cahors, éd. Gérard Defaux et Michel Simonin (Paris : Honoré Champion, 1997), 165-183, ici 177.

29. Jean Bouchet, Chappelet des Princes, cité par Roubaud et par Cornilliat.

30. Nous en donnerons un seul exemple, corrigeant ainsi l'article de François Rigolot qui le donnait pour épique : "Il peut dire + qu'il a vraye richesse »; les autres césures lyriques qu'il relève sont atténuées par un enjambement « à l'italienne » : «Ton Jean le Maire + entre eulx hault colloqué », "Et moy ton père + en joye le receusmes ».

31. Ces deux vers à césure épique devaient aussi échapper à François Rigolot : «O Amaril(le), + moult je m’esmerveillois » et «Et des rui(nes) + fort je m'estonneray » (la diérèse sur " ru-ines » est systématique chez les Marot : 14 occurrences l'attestent chez le père, $20 \mathrm{chez}$ le fils). À la décharge de Rigolot et pour preuve que ces césures étaient sans doute devenues peu maniables (outre le fait qu'on les trouve souvent par paquets, dans des morceaux de bravoure comme chez Cretin), on notera que Bouchet lui-même, alors même qu'il fait preuve d'hypercorrection en reprenant ordinairement les vers épiques enjambant sur une synalèphe (le vers déjà cité « De la justi(ce) + helas certainement » devenant (f. $\left.4 \mathrm{v}^{\circ}\right)$ " Du bien public + helas certainement »), s'est trompé de syllabe en reprenant dans ses Regnars ce vers maladroit : 
décrit comme la relation ambiguë du disciple Marot à un maitre encombrant : il solderait sa dette, un peu de mauvaise grâce, contrairement à Bouchet qui salue un moderne entre les estimables, et le suit radicalement.

\section{L'objet d'un règlement de comptes}

C'est en tout cas bien dans ces termes, d'une discrète liquidation des restes de la poétique médiévale, que l'affaire se joue, et l'hypothèse de Rigolot est très pertinente pour discriminer deux manières de consommer la rupture : « Le grand prédécesseur apparaît ainsi sous un jour étrangement restrictif : un censeur dont l'autorité contrarie singulièrement l'idéal de " liberté formelle » dont Marot se fera par ailleurs le brillant champion $»^{32}$.

Dernier signe de la discrétion du phénomène : chez Fabri, par exemple, on liquide sans en avoir l'air, puisque c'est dans la recette en dix points du Chant-royal (paradoxalement pour cette forme dont la voie d'extinction est bien empruntée en 1521) qu'il proscrit sans autre forme de procès ces types de césures. Entre, pêle-mêle, des recommandations de decorum ou sur la position de l'épithète, sont édictées les règles de la synalèphe pour la césure féminine - tout en même temps que celles de l'alternance des rimes :

Addition selon les facteurs et orateurs modernes [nous soulignons encore] pour bien composer ung champ royal. [...] Item, il doibt eviter les couppes feminines, s'ilz ne sont synalimphees. Item, il doibt user a son champ royal de ligne feminine et puis masculine, ou de masculine et puis feminine. ${ }^{33}$

De public, il n'est encore question qu'indirectement, sous l'angle de la valeur publicitaire du procédé technique. Mais une petite polémique du progrès est tout de même à l'œuvre, implicitement, dans la mesure où se donner à voir dans la modernité de sa pratique n'a pas le même sens selon qu'il s'agisse d'être à la pointe de ce que font les autres — dans cette savante émulation de la

\footnotetext{
«Et mettre ordre + en toutes cours royalles » (lyrique sans synalèphe, 1503, f. E4 v ${ }^{\circ}$ ) devenant « Mectez bon ord(re) + en toutes cours royalles » (épique à l'italienne, 1531, f. 151 vº).

32. Rigolot, " "De peu assez" ", 187.

33. Fabri, Le grand et vray Art, éd. Antoine Héron, 2 : 101.
} 
«Grande Rhétorique » - ou d'être celui qui devance, propose, et vieillit ainsi toujours un peu plus ses prédécesseurs.

Cela nous conduit à un dernier point dans ce rapport de modernisationvieillissement. En termes de pratique éditoriale, sur la crête entre la programmation et la supposition d'un lectorat à prendre, réformer la demande ou s'y conformer n'a pas non plus le même sens. Comme éditeur, Marot a encore un comportement très différencié à l'égard de ce trait d'époque qu'est devenue, sous l'une ou l'autre forme, la césure féminine.

Comme l'a montré Claude Thiry ${ }^{34}$, Clément (le fils du père) édite Villon en lui laissant ses césures médiévales, alors qu'il y est déjà très interventionniste et, surtout, qu'il les corrige dans les œuvres de Jean (son père). Nathalie Hervé ${ }^{35}$ indique que 21 \% des vers du Voyage de Genes de 1507 sont retouchés en 1532 parce qu'ils comportent des césures féminines - peu ou prou la même proportion de $20 \%$ que nous avons relevée chez Bouchet se corrigeant entre 1503 et 1531. Elle cite Mantovani et Defaux, qui analysent ce travail de retouche de Marot :

La vérité est que Clément n'a pas hésité un seul instant à corriger et modifier de façon souvent massive, voire carrément à censurer ce qu'il publiait, à faire en sorte que tout ce qu'il jugeait nécessaire pour léguer à la postérité un témoignage éclatant, et un témoignage concernant non seulement les rares vertus et les incontestables talents d'écriture de son père, mais aussi la façon exemplaire dont lui, son fils, son seul et « vray heritier », avait cru bon d'honorer sa mémoire. ${ }^{36}$

Au-delà des évidentes filiations, les études marotiques convergent pour souligner la propension de Clément Marot à capter sous son nom - masque d'une marque éditoriale qui deviendrait un jour le nom générique d'un «style

34. Voir Claude Thiry, " Marot, éditeur de Villon ", in Villon entre mythe et poésie, éd. Jean Dufournet et Marcel Faure (Paris : Honoré Champion, 2011) : 282-290, ici 282-283, cité par Nathalie Hervé, «De l'œuvre imaginée au recueil imprimé : les recréations métriques dans les éditions de Clément Marot », in Créations d'atelier. L'éditeur et la fabrique de l'œuvre à la Renaissance, éd. Anne Réach-Ngô (Paris : Classiques Garnier, 2014), 150.

35. Hervé, « De l'œuvre imaginée au recueil imprimé », 152.

36. Jean Marot, Les Deux Recueils, éd. Gérard Defaux et Thierry Mantovani (Genève : Droz, 1999), lxvi. 
d'époque $»^{37}-$ la production de ceux dont il prend le sillage (François I ${ }^{\text {er38 }}$ ou son père). Garder son âge métrique à Villon et y laisser le premier Lemaire est tout à fait cohérent avec le fait de retoucher celui du père dont il partage le nom $^{39}$.

Plus discrètement que ne le fera la Pléiade ${ }^{40}$, une génération de poètes cherche à incarner la modernité : on y écrit à rebours des maîtres, parfois de soi-même, en tout cas contre le temps. Mais il s'agit sans doute de se démarquer, plus que de remplacer : Marot entend bien vendre ou sauver, semble-t-il, autant le vieux que le neuf... L'émendation générale qui finit par avoir raison des " coupes féminines » signale en tout cas un emballement : Marot ou Bouchet se modernisent dans les mêmes années où d'autres s'apprêtent volontiers à remotiver cet archaïsme.

Paradoxalement, cette discrète normalisation de la césure du décasyllabe semble avoir eu pour principal écho public des positions radicales relittéralisant le nom du phénomène - cette « couppe femenine » plus évocatrice, voire

37. Sur la notion de «marotisme », voir Isabelle Landy-Houillon, « Autour d'un marqueur stylistique : le marotique ", in Le Génie de la langue française, autour de Marot et La Fontaine, éd. Jean-Charles Monferran (Fontenay : ENS éditions, 1997), 133-145; sur celle de "style d'époque », voir deux études de David Moucaud, respectivement consacrées aux échos de la poésie grivoise et « marotique » entre le $\mathrm{XVI}^{\mathrm{e}}$ et le XVIII ${ }^{\mathrm{e}}$ siècles, et aux «femmes poètes de la Belle-époque » : " (Tout) contre le vers classique, la rencontre des "badins" ", in La Fabrique du XVI siècle au temps des Lumières, éd. Myrtille MéricamBourdet et Catherine Volpilhac-Auger (Paris : Classiques Garnier, sous presse) et "Une décennie "saphique" ? Enquête sur un style d'époque (1900-1909) ", in Les Femmes poètes de la Belle-époque : heurs et malheurs d'un héritage, éd. Wendy Prin-Conti (Paris : Honoré Champion, à paraître).

38. François Rigolot, dans le même article, " "De peu assez" ", 198, salue son " habile[té] " à calquer le nombre de ses rondeaux sur celui de François $\mathrm{I}^{\mathrm{er}}$; mais il lui fait encore concurrence sous le masque de « Prince des Poëtes » dans le jeu de piste des Fleurs de poesie françoyse (1534), ou dans le rôle de celui qui, après Charles d'Orléans ou François, lance à sa (propre) cour, avec le « beau tetin », la règle d'un jeu poétique.

39. Voir les analyses de Gérard Defaux, «Effacer Jehan, \& escrire Clement ", in Clément Marot "Prince des poëtes françois » : 1496-1996, actes du Colloque de Cahors, éd. Gérard Defaux et Michel Simonin (Paris : Honoré Champion, 1997), 81-112 et de François Cornilliat, "Clément Marot et le noble art poétique, ou la preuve par le père ", in "Quand l'ung amy pour l'autre veille ». Mélanges de moyen français offerts à Claude Thiry, éd. Maria Colombo Timelli et Tania van Hemelryck (Turnhout : Brepols, 2008), 195-206 ; voir enfin, bien sûr, Florian Preisig, "Clément Marot éditeur de son père », Travaux de Littérature 14 (2001) : 119-137 et Pascale Chiron, «L'édition des CEuvres de Villon annotée par Clément Marot, ou Comment l'autorité vient au texte », Littératures classiques 64 (2007) : 33-51.

40. Voir l'article de Florence Bonifay dans la deuxième partie de ce numéro. 
suggestive, que ne l'auraient été aucune césure lyrique ou épique. On verra néanmoins que cette ambiguïté recouvre d'autres sources de discorde, aussi symboliques mais moins littérales.

Ce changement technique, qui passe du Puy rouennais (de Fabri) à Marot et à la Cour, donne ainsi lieu à une situation inouïe (si l'on ose dire), en faisant disparaître la " féminine » du vers ancien... sans l'analogie hostile avec laquelle une poésie antiféministe entend en faire une modalité de débat, voire de combat.

\section{Les raisons de la colère : cratylisme et diglossie}

\section{Ambiguïtés misogynes}

«Les mots sont importants $»^{41}$, et le glissement qui conduit la désinence accidentelle du féminin à nommer un point de versification n'est pas anodin. Certes, le phonème incriminé peut garder quelquefois l'apanage de la féminité, et l'on trouverait parfois volontiers des raisons de donner aux césures épiques ou lyriques une quelconque (mais accidentelle) motivation, comme dans ce vers de Jean Marot :

Anne Roy(ne), + des Dames la plus noble ${ }^{42}$

mais un vers voisin viendrait aussitôt en balancer la pertinence :

Gentilhom $(m e)+$ de l'ostel de la Royne ${ }^{43}$

Plus souvent que des noms ou adjectifs susceptibles d'un accord au féminin, se trouvent à cette place des formes verbales ne portant aucune marque de genre.

Pourtant, dans le sillage de la Querelle des femmes depuis le $\mathrm{XV}^{\mathrm{e}}$ siècle, la « couppe femenine » va devenir pour certains le signe de l'une ou l'autre position. Il serait inutile d'aller chercher trop loin un exemple de la misogynie

41. Pour emprunter à Sylvie Tissot et Pierre Tévanian le titre de leur collectif (de critique sociale) voué à clarifier les « euphémismes » et les « occultations » de notre temps : http://lmsi.net et Les Mots sont importants : 2000-2010 (Paris : Libertalia, 2010).

42. Jean Marot, Voyage de Venise (1509), éd. Giovanna Trisolini (Genève : Droz, 1977), v. 955.

43. J. Marot, Voyage, éd. Giovanna Trisolini, v. 1126. 
de rigueur chez les lettrés, qui ne semblent d'abord ni pour ni contre le procédé : au chapitre déjà cité des "vices d'incongruité ", Fabri résume le «feminin parler » à ce « rude et inutille langaige [qui] s'appelle figure de cacosintheton, ou trop feminin et puerille $»^{4}$. Mais le clou est sans doute enfoncé avec cette sorte de figure d'idiotie reprochée à la gent féminine, qui devrait longtemps attendre une revanche proustienne avec le bon docteur Cottard : « une aultre manière de barbare feminin, quant l'en prent mot pour mot assez convenient en prolation, auquel l'en impose nouveau significat par ignorance, comme : "Monsieur, ou est vostre homicide ?" en lieu de "vostre domicille" $»^{45}$. La confusion phonétique est ici moins lexicale (du côté de la paronymie [-omisi-]) qu'elle n'opère à l'échelle du syntagme : le rouennais Fabri, englué dans l'analogie de la "voyelle féminine ", plaque sur une diction féminine une insistance sur la finale, gommant la synalèphe dans une réalisation quadrisyllabique de " votre homicide » qui devient ainsi, comme sous l'effet de la césure lyrique, abusivement équivalent de "votre domicile». Magnanime, Fabri concède cependant que « c'est ung vice qui est a supporter, et especiallement aux femmes qui ont esté avec les clercs, lesquelles ont grand engin et petit entendement $»^{46}$. Lote cite enfin sur ce chapitre un précieux commentaire de Sébillet, du même acabit : "Cest é vulgairement appellé fémenin est aussi fascheus a gouverner qu'une femme, de laquéle il retient le nom $»^{47}$.

Roubaud et Mantovani ont consacré des pages à un véritable mètreétalon en la matière, précisément obsédé par la "guerre de la césure » : Gratien du Pont de Drusac. La synthèse de Roubaud résume assez sa position singulière où l'amuïssement du féminin dans la césure épique prendrait tout son sens :

C'est peut-être à cette [...] obsession misogyne [de Gratien du Pont] qu'il faut associer sa défense de la césure épique; car les théoriciens de la seconde Rhétorique [...] affectent volontiers les «e femenins » d'une quantité plus faible que les syllabes masculines; mais cette appréciation n'est pas que quantitative et technique; elle s'accompagne de toutes les connotations dépréciatives (ou, à l'inverse, chez Bouchet, par exemple, laudatives) du

44. Fabri, Le grand et vray Art, éd. Antoine Héron, 2 : 120.

45. Fabri, Le grand et vray Art, éd. Antoine Héron, $2: 117$.

46. Fabri, Le grand et vray Art, éd. Antoine Héron, $2: 117$.

47. Sébillet, Art poëtique françoys, éd. Francis Goyet, 67 cité par Lote, Histoire du vers français, $4: 24$. 
mot « féminin »; cela fait partie de la querelle des sexes (le $e$ féminin est « $\mathrm{mol}$ » ou « doulx »; il est « foible », « faux », etc.). L'exaltation par Gratien du Pont de la césure épique qui fait d'un $e$ féminin quelque chose de nul et de non compté s'accorde tout à fait avec ses conceptions générales. La césure épique garde quelque chose de la noblesse " masculine " de ses origines : la chanson de geste. ${ }^{48}$

Aussi bifrons que les deux corps du roi, l'e féminin ne serait pas seulement une variable d'ajustement métrique - ce en quoi tout concourt à présenter l'abolition des césures féminines comme un progrès protoclassique - mais il incarnerait encore les vices et les vertus des femmes. Plus précisément encore, si Drusac tolère l'e épique, qui ne fait pas position et donc ne compterait pas, c'est l'e lyrique son contraire qu'il condamne sous un prétexte métrique assez tranché : comme le souligne Véronique Montagne, pour lui, l'e final à la coupe (épique) ou à la rime s'entend (" en toute couppe fault entiere resonance») mais ne se compte pas $^{49}$. Cette négation symbolique et métrique du féminin répond à une véritable obsession chez lui de la réduction des femmes au silence ${ }^{50}$.

Or les deux phénomènes, s'ils touchent à un même problème d'équilibre de la mesure répétée et de réglage métrique, ne sont pas même a priori en concurrence l'un avec l'autre ${ }^{51}$, mais plutôt chacun avec une synalèphe ou une plus ordinaire césure masculine.

48. Roubaud, Impressions de France, 109 ; pour des exemples précis, voir le point que Céline Marcy consacre à la coupe féminine (260-270) dans sa copieuse introduction à Gratien du Pont, Les Controverses des sexes masculin et femenin, éd. Céline Marcy (Paris : Classiques Garnier, 2017), 268.

49. Gratien du Pont, Art et science de rhetoricque metrifiée [Avec la Deffinition de Synalephe, pour les Termes qui doibvent synalepher, et de leurs Exceptions. Les Raysons pourquoy synalephent et pourquoy non. Choses encore non specifiées ny illucydées par les Autheurs qui ont composé sur ledit Art, en langue Françoyse, jusques au present] (1539), éd. Véronique Montagne (Paris : Classiques Garnier, 2012), 38-39.

50. Comme le montre Diane S. Wood («Gratien du Pont et la Querelle des femmes, 1534-1541» [titre de la communication initiale : "Toulouse et Paris : deux pôles de la Querelle des femmes : 1534-1541 »], in Revisiter la "Querelle des femmes”. Discours sur l'égalité/inégalité des sexes, de 1400 à 1600, éd. Armel Dubois-Nayt, Nicole Dufournaud et Anne Paupert (Saint-Étienne : Publications universitaires de SaintÉtienne, 2013), 89), l'ostensible amuïssement des césures féminines extranuméraires n'en est qu'une démonstration. Son jeu d'échecs misogyne pourrait également répondre au dispositif paritaire du jeu de Dames...

51. Martinon, «Études sur le vers français », 64, reproche à des «poètes amateurs » au nombre desquels il tient Marguerite de Navarre, de recourir « concurremment » aux deux types de césure, suivant en cela 
Sébillet, dans la caractérisation très littérale qu'il leur donne aussi, ne s'y trompe pas : "L'é fémenin se congnoistra plus aisément conféré avecques son masle : car il n'ha que demy son, et est autrement tant mol et imbécille, que se trouvant en fin de mot et de syllabe, tombe tout plat, et ne touche que peu l'aureille $»^{52}$. Il parle encore d'un « mol et flac son $»^{53}$ et signale qu' il n'est " pour rien cont[é] [en la fin du vers], non plus que lés femmes en guerres et autres importans affaires, pour la mollesse de cest é fémenin $»^{54}$.

Le plus frappant est toutefois qu’après avoir ainsi palabré sur la « coupe féminine » des $e$ de fin de vers, Sébillet décrit la « coupe féminine » à la césure... en décrivant exclusivement des cas de césures enjambantes, qui « synalèphent » parfaitement. Césures épique et lyrique ne lui sont plus connues, ou plutôt néglige-t-il même de les mentionner ${ }^{55}$.

\section{Des enjeux plus discrets}

Pour terminer ce parcours en cherchant à débrouiller l'écran de fumée émanant, sous l'abstraction misogyne, d'un feu de tout bois (de fond comme

\footnotetext{
" une logique fort contestable » du fait de leurs origines éloignées. Mais un tel raisonnement ne l'est guère moins, qui proscrirait aussi bien l'emploi de deux mètres dans une même pièce... ; on consultera toutefois avec agrément sa mise en récit.
}

52. Sébillet, Art poëtique françoys, éd. Francis Goyet, 68.

53. La discrète équivoque qu'exhibe l'antéposition, inhabituelle entre monosyllabes, pourrait être empruntée aux "propos des bienyvres » du Gargantua. Rabelais s'en délecte par quatre fois dans ce seul chapitre jusqu'à l'expliciter tout à fait : «bouteille est fermée à bouchon, et flac con [en 1538 ; flaccon en 1542] à vitz " (Les Cinq Livres, éd. Jean Céard, Gérard Defaux et Michel Simonin [Paris : Librairie générale française, La Pochothèque, 1994], 33). Nous remercions l'œil alerte et l'oreille affûtée de nos éditeurs pour cette suggestion.

54. Sébillet, Art poëtique françoys, éd. Francis Goyet, 69 ; Véronique Montagne développe avec précision la question phonétique dans son introduction (Gratien du Pont, Art et science, 32-36), tandis que les dérives au long cours de cette sexuation des phonèmes sont bien synthétisées dans Christine Planté, "De quelques discours sur l'e muet », in Nature, langue, discours, éd. Merete Stistrup Jensen (Lyon : Presses universitaires de Lyon, 2001), 47-63.

55. Francis Goyet signale (Sébillet, Art poëtique françoys, 155, note 75 de la page 73) les exemples non pertinents de Sébillet, pour qui, dans le «mauvais vers " qu'il a plutôt mal lu " Puisque l'âme est + en ce corps descendue ", l'e d'« âme » pourrait selon lui être synaléphé ou « laissé entier » (73); ce qui le dérange dans ce vers parfaitement «métrique » au regard de sa césure tient plutôt à la coupe, doucement italianisante, sur l'auxiliaire. 
de forme), nous reprendrons à Roubaud l'hypothèse qu'il esquisse ${ }^{56}$ sans la développer, d'un "parti toulousain » dont Gratien Du Pont et Jean Du Pré, autre militant de la césure épique contre la lyrique, seraient les fers de lance. Kastner identifiait déjà la nette latence dans le changement à la césure, entre les poètes de la cour de France et « ceux du Hainaut ${ }^{57}$, et Claude Thiry soulignait au plus long cours leurs cultures poétiques différentes, observant en termes de " milieux littéraires " deux " obédiences », bourguignonne (très défavorable à la coupe épique et à cet $-e$ qui traîne) et française ${ }^{58}$. Dans ces années $1490-$ 1510, l'obédience bourguignonne prend le dessus, via Rouen, avec l'élagage dont Lemaire, comme l'a suggéré Thiry, n'est finalement qu'un adepte tardif et systématique.

Il semble de surcroît que la distance des cultures linguistiques s'y fasse sentir. Dans sa réforme silencieuse, l'« abolition des coupes féminines » est avant tout une régulation de la langue poétique : métrique au premier chef, puisqu'elle a pour conséquence stricte de normaliser la longueur de la première séquence du décasyllabe, elle est aussi phonétique et, à ce titre, un écho de la prononciation normée. Les finales féminines sont un lieu privilégié de la variation diatopique, ou pour le dire autrement la chambre d'écho des accents méridionaux qui, aujourd'hui comme alors, font davantage entendre les posttoniques. Si certaines restitutions de la « langue baroque» favorisent l'hypothèse selon laquelle l'amuïssement des finales féminines dans la déclamation ne serait pas achevé au début du XVII ${ }^{\mathrm{e}}$ siècle ${ }^{59}$, on peut raisonnablement envisager qu'un

56. Roubaud, Impressions de France, 110 ; Benoît de Cornulier y songe de manière critique quant à la rime féminine, dans « Types de césure, ou plutôt manières de rythmer le vers composé ", L'Information grammaticale 121 (2009) : 21-27, 25 : « Il ne faut pas confondre le simple fait de prononcer l'e féminin final comme encore aujourd'hui dans le Midi de la France et le fait, s'il est prononcé, d'en faire l'appui rythmique comme à la césure dite "lyrique", ce qui n'est conforme à la prosodie française ni du Nord, ni du Midi. "

57. Kastner, «Les grands rhétoriqueurs », 291.

58. Voir Claude Thiry, « De la césure féminine comme adjuvant à la localisation des textes poétiques en moyen français ", Le Moyen français. Le traitement du texte : édition, apparat critique, glossaire, traitement électronique, actes du IX colloque international sur le moyen français (1997), éd. Claude Buridant (Strasbourg : Presses universitaires de Strasbourg, 2000), 185-208 et Claude Thiry, " Rhétoriqueurs de Bourgogne, Rhétoriqueurs de France : convergences, divergences ? ", in Rhetoric Rhétoriqueurs Rederijkers, actes du Colloque d'Amsterdam (1994), éd. Jelle Koopmans, Mark A. Meadow, Kees Meerhoff et Marijke Spies (Amsterdam : Rodopi, 1995), 101-116.

59. Voir notamment Eugène Greene, La Parole baroque (Paris : Desclée de Brouwer, 2001). 
écart, même par une simple translation, distinguait des dictions toulousaines, par exemple, d'un idéal plus sec et plus arithmétique où une syllabe doit faire ou ne pas faire position. À la suite de Véronique Montagne, nous postulons qu'une tendance se fait jour dans le rapport différencié entre les pratiques des poètes du Nord et de France, propre à favoriser la persistance (et la défense) des coupes féminines, qu'elles soient comptables (lyriques) ou non (épiques) ${ }^{60}$. Cet « $e$ qui traîne » que le normand Fabri imputait à une faiblesse féminine, est d'autant plus sensible au Sud où des finales féminines surnuméraires, à la césure comme à la rime, menacent une métrique que les artes contemporains continuent de régler ${ }^{61}$.

Plus encore, il conviendrait d'envisager cette résistance poétique sous l'angle d'une dissidence au triomphe de la langue royale. Notons, parmi les précédents à l'édit de Villers-Cotterêts, que l'ordonnance de 1510 (Louis XII) donnant la primauté des «langues du peuple » sur le latin n’est appliquée au Languedoc que bien tard (en 1535, avec l'ordonnance d'Is-sur-Tille). Peu avant le « langage maternel français » du fameux édit de 1539, et contrairement aux ordonnances précédentes qui envisagent la "langue du peuple», celle d'Is-surTille maintient une ambivalente alternative où s'entend encore une résistance contre la volonté royale : «doresnavant tous les proces criminels et les enquestes seront faictz en françoys ou a tout le moins en vulgaire dudict pays $»^{62}$.

L'abolition (relativement) définitive des coupes féminines dans les années 1510 coïnciderait peut-être moins avec un sursaut de la querelle des femmes,

60. En ayant à l'esprit les réserves de Cornulier sur la valeur arithmétique " nette » d'une diction méridionale, on se reportera à la démonstration de Véronique Montagne, in Gratien du Pont, Art et science, 34-35, suivant Wilhelm Theodor Elwert, qui oppose une " prononciation durable et reconnue pour typiquement méridionale ", défendue par Drusac, à une " évolution prosodique standard, qui est celle du Nord de la France, laquelle a amené un amuïssement presque total du $e$ dit "caduc" avant le début du XVI ${ }^{e}$ siècle et “total dans le langage quotidien à partir du premier tiers du XVII siècle" ».

61. Quoiqu'il soit courant jusque tard dans le siècle de considérer les vers féminins comme des vers impairs, il est difficile de ne pas voir Drusac protester contre cet usage lorsqu'un huitain de L'Art et science de rhetoricque métriffiée donne position à des rimes féminines en systématisant sous le nom d'« heptasyllabes » des vers brefs multipliant les féminines à la (dé)faveur de définitions dégradantes, à l'exact inverse du vers de sirventois, tels que «Femme : ce n'est qu'ordure ». Diane S. Wood, « Gratien du Pont et la Querelle des femmes », 85, signale et cite ce huitain.

62. Cité par Douglas A. Kibbee, "Louis Meigret lyonnais et les politiques de la langue française à la Renaissance ", in Lyon et l'illustration de la langue française à la Renaissance, éd. Gérard Defaux et Bernard Colombat (Lyon : ENS Éditions, 2003), 63-76, ici 67. 
que, plus silencieusement, avec l'étape la plus flamboyante de l'arasement linguistique et poétique en marche. À rebours de ce mouvement, la somme misogyne que Drusac prétend mettre en signes en systématisant le procédé d'une césure épique antiféminine, ne pourrait-elle pas dès lors se doubler d'une autre forme de résistance, aussi gasconnante que viriliste ? En parallèle d'une traditionnelle négociation entre le français et son voisin transalpin ${ }^{63}$, la conflictualité a beau être gommée de certaines velléités plurilingues au sein du royaume, comme chez Jean Du Pré ${ }^{64}$ en 1534 ou en 1553 chez Bernard du Poey, elle est chez lui très sensible et se prolonge en 1555 dans le recueil de $\mathrm{La}$ Requeste faicte et baillee par les Dames de la ville de Tolose ${ }^{65}$, " Dames » d'ici et de là portant haut fiertés et dédains.

$\mathrm{Si}$, dans un cas comme dans l'autre, le fond est antiféministe, la forme et le vecteur sont aussi des provocations « régionalistes ", localisées. Diane S. Wood, qui voit en Drusac un « médiocre poète de province » souligne cet effet polarisateur sur les lectorats-cibles :

Son influence dans sa ville natale, sa grande capacité à inventer des vers antiféministes et le ton railleur de son œuvre enflammèrent les lecteurs et lectrices de Lyon et de Paris, et servirent à attiser la Querelle. [...] L'édition toulousaine d'une œuvre littéraire par le responsable des Jeux floraux avait sans doute pour ambition initiale de séduire le lectorat local, mais le nombre de rééditions ailleurs en France atteste d'un intérêt considérable suscité par le texte dans le reste du pays. ${ }^{66}$

63. Laquelle se négocie en permanence dans les représentations poétiques, entre la Concorde des deux langages de Lemaire en 1511 et la Deffence et Illustration de la langue françoyse de Du Bellay en 1549.

64. Montagne, in Gratien du Pont, Art et science, 43, cite Le Palais des nobles dames de $1534:$ : [...] Françoys, plains de doctrine, / L'ont en hault pris ; Tholouse la defend / [...] à tous adhère, et ne veulx point querelle».

65. La Requeste faicte et baillee par les dames de la ville de Tolose, éd. Jean-François Courouau et Philippe Gardy (Toulouse : Presses universitaires du Mirail, 2003) ; que Michel Jourde soit ici remercié pour ses analyses inspirantes, recueillies dans le présent volume. Les césures épiques des décasyllabes sont spectaculairement nombreuses dans les pièces en français de ce recueil (50 pour 152 vers), comme dans les pièces " gasconnes" (114 pour 256 vers); dans Les Controverses des sexes masculin et femenin de Drusac, les césures épiques se trouvent dans la même proportion que dans ce recueil : au-delà de $30 \%$ (4800 des 14500 vers) ; pour un quart, elles synalèphent à l'italienne.

66. Diane S. Wood, « Gratien du Pont et la Querelle des femmes », 79-80. 
Cet antiféminisme de Drusac, pourtant forcené quant au discours, connaît une autre limite dans sa mise en forme. Fabri avait beau sexualiser la coupe féminine et la rejeter, il encourageait l'alternance des rimes en genre ${ }^{67}$. Or, si par inertie ou par conservatisme, les poètes du Puy n'y sacrifiaient guère ${ }^{68}$, Drusac l'applique quant à lui malgré son aversion radicale du féminin. Sur ce point précis, il est plus défavorable à voir disparaître un $e$ post-tonique qu'il a tout de même pris soin de disqualifier, qu'à concéder le terrain d'une certaine équité formelle.

On pourrait enfin rapprocher cette éviction imparfaitement consensuelle $\mathrm{du}$ rapport linguistique bien différent qu'entretient la poésie française des années 1510-1550 avec la vogue de l'italien, qu'elle intègre plus volontiers. Plus visible (et finalement audible) qu'une finale surnuméraire devant une consonne, la synalèphe "épique ", par exemple, comme de plus rares et contestables césures (masculines) "à l'italienne ${ }^{69}$, font chevaucher un mot par la césure, sans altérer l'intégrité de l'hémistiche. Si une fine synthèse des italianismes poétiques du premier $\mathrm{XVI}^{\mathrm{e}}$ siècle reste à faire, on observera combien l'histoire reste plus discrète sur les réticences gasconnes que sur la plaisante anecdote de la "règle de Marot » touchant l'accord des participes, à l'italienne. Cette guerre larvée et complexe de la césure puis sa dernière bataille menée par Drusac, outrancière jusque dans sa versification, recouvrent pour partie le jeu différencié des influences sur la poésie française à l'heure de son illustration : dans un cas, on exhibe un féminin compté pour nul (qu'impose le signe métrique et que surdétermine une trace phono-dialectale), de l'autre on normalise une plus discrète défense de l'égalité devant l'accord. Autres temps, autres mours, vraiment?

67. On a pu mettre en relation ce procédé plutôt propice à l'égalité des sexes, avec la création contemporaine de cette autre artificialisation du signe qu'est l'allégorique jeu de Dames (voir Kathleen Wilson-Chevalier, "Art Patronage and Women in the Orbit of Francis I ", Renaissance Studies 16.4 [2002] : 474-524).

68. Voir Hüe, La Poésie palinodique, 900 : «En 1544, un quart des chants royaux persiste à ne pas alterner rimes masculines et féminines, et lorsque, vers 1530, Des Arpens correspond avec Bouchet, l'alternance lui semble inconnue ».

69. Voir sur ce point l'étude de Benoît de Cornulier, « Sébillet contre l'italianisme métrique. À propos de césure et de sonnet français vers 1548 », Revue d'histoire littéraire de la France 105 (2005) : 190-199. 


\section{Conclusion}

«Comme si le vers du règne de François $\mathrm{I}^{\mathrm{er}}$ se devait d'être un vers nouveau » ${ }^{70}$, écrit Roubaud au sujet de cette réforme silencieuse des années 1510. Au profit de jeunes et de moins jeunes premiers (de cour), l'heure est en effet à une mise aux normes, d'abord linguistique, dont la «bataille de la césure » pourrait bien être l'un des moyens, plus discrètement, plus insidieusement que d'une allégorique censure des femmes dont on va bientôt l'habiller.

Elle archaïse instantanément la coupe lyrique, qui devient dès lors une borne générationnelle, de précieux secours pour la datation de certaines pièces (chez Clément Marot, Marguerite de Navarre ou encore Jean Bouchet) et fait de la coupe épique une plus grande infraction à la métrique «nombreuse », c'està-dire syllabique, qui se grammatise plus avant au début du siècle. La résistance que lui donnent respectivement, pour des raisons qui semblent diamétralement opposées, Marguerite de Navarre et Gratien du Pont de Drusac ${ }^{71}$, est plus difficile à cerner. Si le phénomène doit être également un marqueur, il est plus visible encore que celui de la césure lyrique. Des historiens de la phonétique seraient mieux placés pour évaluer les choix à l'œuvre, chez ceux qui « mangeraient » volontiers les finales féminines au point de les élider à cette position, ou chez ceux qui au contraire y trouveraient le prétexte de leur donner le corps auquel aspire leur accent. Nous ne trancherons pas pour Marguerite, mais sommes tenté d'avancer la seconde hypothèse pour le camp de Drusac, sinon pour y voir une altération métrique, du moins une tendance déclamatoire et, partant, le signe d'une rébellion autant que d'une esthétique.

Le bruit d'un Drusac ne donne pourtant lieu à aucune véritable guerre de la césure, comme l'orthographe s'apprête à en connaître une. Nul Drusac ne s'érige davantage contre une autre règle nouvelle, qui concerne également des finales féminines et consiste en l'accord des participes dont l'objet est antéposé : ouvertement imité de l'italien, le principe est vite admis comme une règle, et d'une manière assurément moins polémique - les réticences s'y expriment du moins sans heurts - que n'en seront certaines réminiscences au $\mathrm{XXI}^{\mathrm{e}}$ siècle $^{72} \ldots$

70. Roubaud, Impressions de France, 101.

71. Martinon, «Études sur le vers français », prend les deux pour exemples.

72. La récente et vive émotion autour d'accords et de stratégies graphiques visant à inclure dans la norme linguistique l'idée d'une parité des sexes montre une fois de plus combien l'écriture enregistre les étapes d'une éternelle « querelle des femmes ». Aussi peut-on s'étonner qu'à la même époque on puisse encore 
L'e, caduc ou comptable, du participe, ou de la fin des mesures métriques, fait l'objet d'un arbitrage du signe qui n'a finalement pas seulement à voir avec la question féminine. Mais si la règle d'accord comme celle de l'alternance rimique $^{73}$ peuvent être, à la discrétion des poètes, encore lentement adoptées et donner le change d'un contrepoint, l'ordre métrique impose sans conteste à la césure une immédiate masculinisation ou sa réduction synaléphique.

Il resterait à creuser cette question de l'étouffement diglossique en étudiant peut-être hors des seuls cas de Drusac et de Du Pré les productions des Jeux floraux toulousains entre 1490 et 1510 - dont l'académie connaît en 1512-1513 une troublante «francisation » en devenant «Collège de Rhétorique » - et dont la forme-reine est le Chant-Royal, dans lequel précisément Fabri a condamné à Rouen les coupes alternatives. Il semble temps de mesurer la force de partition linguistique de ce changement poétique collectif.

«Il y avait comme deux camps. Il fallait, pour trancher la question, un poète de génie qui s'imposât ", écrit Kastner ${ }^{74}$. Le " cadurcien » vagabond qu'était Clément Marot était né d'un père rouennais passé en Bretagne et du parti des Dames, qui lui avait transmis « la paternelle / Langue françoyse, es grands Courts estimée, / Laquelle enfin quelque peu s'est lymée, / Suyvant le Roy Françoys, premier du nom $»^{75}$. Celui dont la Cour fut la «maistresse

contester la « règle de Marot » qui ne semblait plus menacer, depuis son paramétrage par Vaugelas et malgré son artifice originel, qu’une poignée d'écoliers récalcitrants : « En toute la Grammaire Françoise, il n'est rien de plus important, ny de plus ignoré ", écrivait-il en 1647 (Remarques sur la langue françoise, cité par Nathalie Fournier, Grammaire du français classique [Paris : Belin, 2002], 326, $\$ 458 \mathrm{~b}$ et Maurice Grevisse et André Goose, Le Bon Usage [Bruxelles : De Boeck, 14éd., 2007], 1169, \$943). Que l’on en condamne la complexité, ou que l'on voie en elle un outil de distinction sociolinguistique (voir Luce Petitjean, "Un vieux casse-tête : l'accord du participe passé », Mots. Les langages du politique 28 [1991] : 70-85), il faudrait surtout en suivre les premières motivations, qui nous ramènent aux trois aspects que nous venons d'examiner sur les coupes féminines...

73. L'allongement du vers par la rime féminine, travaillé sur des actes entiers dans le théâtre de Jodelle par exemple, fait lui aussi l'objet d'une normalisation : il est réglé par une parité arithmétique et neutralisé par l'alternance. Pour une perspective historique sur cette dernière dans la génération de Gringore et Bouchet, voir Léon Émile Kastner, «L'alternance des rimes depuis Octovien de Saint-Gelais jusqu'à Ronsard », Revue des langues romanes 47 (1904) : 336-347.

74. Kastner, «Les grands rhétoriqueurs », 294.

75. Clément Marot, "L’Enfer » [1526], Euvres poétiques 2, éd. Gérard Defaux (Paris : Bordas, 1992), 30-31. On consultera avantageusement, dans cette perspective, les pages de Gilles Guilhem Couffignal, qui prend Marot pour exemple d'une diglossie pacifiée : "Est-ce pas ainsi que je parle ?” : la langue à 
d'escolle " synthétise ces conflits et incarne leurs apaisements ; ce faisant, il nous renvoie aussi au triomphe de la sphère royale en matière de domination linguistique.

Les " coupes féminines " étaient donc restées en usage comme un ornement mal réglé chez les Rhétoriqueurs, et vouées à disparaitre au profit d'une mesure plus claire ; mais leur rapide disparition, en suscitant étrangement peu de protestations dont nous ayons la trace, sinon une opportuniste tentative, est l'occasion d'un certain nombre de discrets règlements de comptes : avec une pratique ancienne devenue inadaptée (ces deux césures étant assez contradictoires au sein d'un même système) ; par ricochet, comme rebond allégorique des débats misogynes et philogynes de la Querelle ; enfin, peutêtre, avec le spectre d'une pluralité des tons et des atones dans une production poétique que la cour des derniers Valois avait voulu centraliser...

l'œuvre chez Pey de Garros et Montaigne (thèse de doctorat, Université Toulouse 2, 2014), notamment $176-180$. 